\title{
MÉTAPHORE ET NARRATIVITÉ
}

\author{
Philippe Marion ${ }^{1}$
}

Métaphore et récit. De prime abord, associer ainsi les deux concepts peut paraître étonnant. Ou quelque peu forcé. Quoi de commun, en effet, entre cette figure de substitution régie par un principe d'analogie et la reconfiguration organisée d'événements réels ou imaginaires. Quoi de commun, si ce n'est l'utilisation narrativement anecdotique de quelques métaphores, en vue d'agrémenter le "sérieux" et l'urgence logique du récit en cours. Au sein de l'espace réservé des descriptions, par exemple. S'agissant de trouver des complicités rhétoriques, on se tournerait plus spontanément vers la métonymie. Si du moins, à la suite de Jakobson, on accepte de redéfinir métaphore et métonymie non pas tant comme figures que comme processus. Comme une alternative fondamentale dans les modes d'organisation du langage. Axe des contiguïtés, chaîne de glissements de cause à effet, onde de contagion logique, effet de dominos lorsqu'après une impulsion initiale ces dominos s'entraînent mutuellement dans leur chute... Dès lors qu'on se représente de telle façon (par de telles métaphores...) le processus métonymique, l'air de famille avec le récit ne fait plus de doute.

1 Professeur au Département de communication de l'Université catholique de Louvain.

Recherches en communication, $\mathrm{n}^{\circ} 2$, (1994). 
Pourtant les propos qui suivent voudraient souligner quelques passerelles entre métaphore et récit. Non par jeu de spéculations intellectuelles -que l'on s'empare de deux concepts et dès lors qu'ils sont des concepts- on peut toujours y trouver matière à comparaison.; mais parce que la métaphore me semble permettre une meilleure compréhension du fonctionnement narratif. Aussi parce que nombre de récits "postmodernes" ne trouvent leur plein épanouissement, quel que soit leur support médiatique, que par l'aménagement de parcours métaphoriques. La métaphore peut même s'y révéler au lecteur ou au spectateur en tant qu'objet d'une quête spécifique. Objet de désir d'un lecteur modèle $e^{1}$ cherchant activement des voies d'interprétation.

Après une première mise en contact conceptuelle du récit et de la métaphore, je tenterai de montrer différentes manières dont la narration peut tirer profit, s'appuyer et même se construire sur une construction métaphorique. Situer la métaphore face au récit, c'est forcément aussi la situer dans la réalisation expressive et médiatique de ces récits. Pas de fable sans la médiation d'un matériau d'expression choisi. A cette fin, différents champs médiatiques seront abordés tout en réservant une place importante à la bande dessinée.

\section{La métaphore ressemble-t-elle au récit?}

En tant que processus, la mise en métaphore et la mise en récit ne sont pas sans point commun. Selon la définition structurale classique (Greimas, Barthes, Bremond...), le récit procède de la rupture délibérée d'un équilibre initial tendant à se résoudre dans un nouvel équilibre. Ou, pour reprendre la formulation de Kristeva, d'une «suspension de la disjonction initiale»" ${ }^{2}$ Le cœur du récit -sa "boîte noire"- ne peut battre que dans l'espace-temps organisé de cette disjonction suspendue. Telle est la nature profondément disjonctive du récit.

1 Pour reprendre l'expression rendue célèbre par Umberto Eco.

2 Julia Kristeva, Semeiotikè, Paris, Seuil, Points, 1969, p. 66. 


\section{Rupture d'équilibre}

Construire une métaphore, c'est chercher puis choisir, dans une catégorie de signes, une unité qui ressemble à celle que l'on veut remplacer afin de la mieux représenter. C'est, pour mieux la représenter, remplacer une unité par une autre qui lui ressemble quant au sens, mais que l'on recherche et choisit dans une autre catégorie sémantique. Ainsi, la métaphore utilise souvent un terme concret pour représenter une idée abstraite ou s'inscrivant dans un contexte abstrait. Cette transposition du "propre" dans le "figuré" s'opérant sur la base d'une relation d'analogie. La "fuite" ou l"'écoulement" du temps, le "sommet" d'une carrière, le "carrefour" d'une vie, être sur une "pente glissante", etc.

Dans un certain sens, la métaphore est elle aussi une rupture, une transgression de frontière entre deux univers de référence. $\mathrm{Ne}$ craignant pas l'amalgame, la métaphore procède donc d'une rupture d'étanchéité entre deux catégories normalement séparées. Deux catégories placées "normalement" -dans ce qu'on pourrait appeler un degré zéro de l'expression-représentation- en situation d'équilibre initial dans la disjonction. Comme le récit, la métaphore manifeste donc à sa façon la perturbation d'un équilibre initial et la suspension d'une disjonction (notamment cette disjonction traditionnelle qui sépare le concret de l'abstrait). Tel est le sens de cette "impertinence" étudiée par Paul Ricœur ${ }^{1}$ : la métaphore est impertinente parce qu'elle révèle l'utilisation non appropriée d'un signe dans un contexte sémantique et un environnement référentiel qui lui est étranger. Construite sur une méprise volontaire, une transgression catégoriale, la métaphore est donc un déséquilibre littéral -littéralement, un déséquilibre- par surgissement d'un élément étranger:

"Le mot métaphorique vient à la place d'un mot non métaphorique qu'on aurait pu employer (si du moins il existe); il est alors doublement étranger, par emprunt d'un mot présent et par substitution à un mot absent"2.

Parler du jacassement d'une personne, c'est de façon impertinente (à plus d'un titre...) lui découvrir une caractéristique sonore attachée spécifiquement à la pie. Dans la métaphore «le sens emprunté s'op-

1 Voir Paul RICcEur, La métaphore vive, Paris, Seuil, 1975.

2 Ibidem, p. 28-29. 
pose au sens propre» ${ }^{1}$. Et cet emprunt, cette transposition, bref cette "epiphora" étudiée jadis par Aristote signifie la rupture d'un ordre logico-sémantique. Déséquilibre dont la dynamique profonde n'est pas sans rapport avec celle qui génère le récit. Construite sur la transgression de l'ordre catégorial, la métaphore re-décrit la "réalité", ou plutôt elle reformule la représentation d'un réel. Comme le récit re-configure le substrat événementiel réel ou imaginaire.

\section{Transformation, modification, transposition}

Le. récit n'est pas pensable sans un principe de transformation, c'est-à-dire un dosage dans la succession (le temps) d'éléments communs et d'éléments distincts. Commentant les travaux de plusieurs narratologues tels Bremond et Todorov, Gaudreault montre bien que succession et transformation sont les conditions essentielles pour fonder la narrativité d'un énoncé. Il ajoute que la transformation ne doit pas être comprise comme substitution mais bien davantage comme modification ${ }^{2}$. Dans leur succession, les éléments du récit ne doivent être ni tout à fait semblables, ni tout à fait différents. Il faut cette altérité et cette permanence, «sinon la narrativité n'arrive pas à se manifestern ${ }^{3}$. Selon Todorov, la narrativité ne peut d'ailleurs se constituer que dans la tension de ces deux catégories formelles:

\footnotetext{
"Si les prédicats ne changent pas, nous sommes en deçà du récit, dans l'immobilité du psittacisme; mais s'ils ne se ressemblent pas, nous nous trouvons au-delà du récit, dans un reportage idéal, tout forgé de différences" 4 .
}

La métaphore s'appuie elle aussi sur une dynamique de transformation. Or qu'est-ce qu'une transformation sinon une synthèse de ressemblances et de différences? D'une part, on y savoure un écart, une transgression, une différence: cet emprunt plus ou moins inattendu à une catégorie sémantique "étrangère". D'autre part, on doit y reconnaître une filiation, une permanence par la médiation d'une ressemblance. Si la transgression est trop intense, si se perd le fil conducteur

1 Ibidem, p. 25.

2 André Gaudreault, Du litttéraire au filmique, Paris, Méridiens Klincksieck, 1989, p. 45.

3 Ibidem, p. 46.

4 Tzvetan Todorov, Poétique de la prose, Paris, Seuil, 1971, p. 240. 
de la transposition par analogie, le destinataire se heurtera à l'incompréhension et butera sur un effet de jargon hermétique, incongru ou gratuit. Ou alors, il croira se trouver face à une énigme. Face au Sphinx. Mais ne devient pas CEdipe qui veut.

\section{Limites de la ressemblance}

Ce qui précède propose une définition assez minimaliste du récit. Par trop laxiste, reprocheront certains. Ce qui, du reste, confirme la nécessité de distinguer récit et narrativité, ce dernier concept renvoyant à une virtualité, à une propension narrative due à certaines caractéristiques d'un énoncé. J'y reviendrai plus loin. Ce reproche d'une définition trop large du récit a d'ailleurs suscité nombre de controverses. Ainsi par exemple, Odin ne partage pas la manière dont Gaudreault pose la transformation comme condition nécessaire et suffisante du récit: «car il est bien clair, explique-t-il, qu'il peut exister des transformations qui ne mettent pas en cuvre la temporalisation: par exemple la métaphore» ${ }^{1}$. Rappelant l'importance de penser "modification" plutôt que "transformation", Gaudreault ajoute: "quand une métaphore fonctionne comme une modification de quelque chose donné dans un premier temps (ce qui n'est pas toujours le cas), elle implique dans ce cas une forme de temporalisation ${ }^{2}$. Ce qui conforte implicitement ce que je suggérais ci-dessus: métaphore et récit relèveraient partiellement d'une même dynamique de temporalisation attachée à la "modification".

Si l'on admet cette ressemblance dans la temporalité, on gagnerait sans doute à la rapprocher d'une autre condition nécessaire à l'émergence du récit: la mise-en-intrigue. Ce concept proposé par Ricœur vient contrecarrer les accusations de laxisme à l'égard des conditions minimales de succession et de transformation. La mise-enintrigue, c'est-à-dire un "acte configurant dans le temps", ce qui permet d'articuler succession et transformation dans le contexte d'une histoire racontée.

Quant à la métaphore, si elle semble obéir elle aussi à un processus de temporalisation, elle n'atteint jamais explicitement la spécificité

\footnotetext{
1 Roger ODIN, "Du spectateur fictionnalisant au nouveau spectateur", in Iris, n8, avril 1988.

2 André Gaudreault, op.cit., p. 46.
} 
narrative d'une mise en intrigue. De plus, cette temporalisation liée au travail de transformation antérieure est toujours déjà jouée au moment de l'énonciation. Du moins si l'on cantonne la métaphore dans les limites de la catégorie rhétorique traditionnelle des figures de substitution (combinant les opérations fondamentales de suppression et d'adjonction). Contrairement au récit qui ne parvient à survivre sans agencements temporels exprimés, la métaphore porte en elle l'effacement de la transformation qui l'a fait naître. Surgissant de l'ellipse de la comparaison qui l'a générée, la métaphore apparaît comme un résultat, un "prêt à porter". Ou plutôt, comme un prêt à communiquer.

La différence pragmatique avec le récit me paraît, à ce titre, essentielle. La transposition-transformation propre au mouvement métaphorique, c'est le récepteur qui doit lui rendre sa consistance. Le temps de 1"epiphora" aristotélicienne, le temps du transfert catégorial, c'est le destinataire qui doit l'appréhender et le réactualiser. S'il existe une temporalisation dans la métaphore, elle ne peut être reçue que dans le repérage d'une modification. La saisie et le re-parcours du cheminement analogique qui constitue sa genèse sur le plan de la production, ce qui permet de l'adapter au contexte étranger dans lequel elle affleure.

Quant à la notion commune de rupture d'équilibre évoquée cidessus, elle ne peut non plus rapprocher totalement métaphore et récit. La métaphore ne s'organise pas autour de la recherche d'un équilibre final. La rupture d'équilibre y est dissimulée. Résultat d'une opération antérieure à l'énonciation, elle s'y présente déjà avec les attributs et l'évidence péremptoire d'un nouvel équilibre. Charge au destinataire d'évaluer la trajectoire de rupture amenant au résultat métaphorique. Cette responsabilité du récepteur est en outre la source de son plaisir. Plaisir de découvrir une trace de style, c'est-à-dire la trace du sujet unique qui fut capable d'opérer de telles substitutions analogiques, d'avoir osé de telles fantaisies transgressives.

\section{Métaphore et transparence}

La réception d'un message métaphorique pose en outre l'intéressante question stylistique des écarts ressentis. En effet, bon nombre de métaphores (verbales, iconiques, ou scripto-visuelles...) sont passablement usées. Intégrées dans l'évidence d'un code culturel, elles ne 
sont plus perçues comme métaphoriques. L'effet de transposition n'apparaît plus dans une expression comme "le sommet d'une carrière". Plus vive est la métaphore, plus son "impertinence" est perçue comme une figure, plus elle invite le destinataire à reconstruire le cheminement absent de la transformation, à mesurer la stratégie de la transposition. Plus la métaphore est vive, plus on peut l'évaluer comme une "prise de risque" expressive de l'énonciateur. Plus elle est vive, plus elle est opaque dans sa dimension "sui-réflexive"l.

Mais les choses ne sont sans doute pas si claires. Et la position inverse peut facilement être tenue. Les métaphores usées, lorsqu'elles le sont vraiment trop, comme "le blanc manteau" pour exprimer la neige hivernale, réintroduisent une opacité, dans le sens d'un bruit de communication. Lorsque la formule expressive apparaît comme cliché saturé, elle se révèle surtout comme formule, c'est-à-dire comme médiation délibérée, comme stratégie stylistique qui ne parvient pas à effacer les traces de son labeur. Ni surtout celles d'un effet trop manifestement recherché. L'impression de métaphore usée, ressentie par le récepteur s'associe souvent à la mise à nu de l'intentionnalité vaine de produire un effet, ce qui est incompatible avec toute séduction rhétorique. Dans cette optique, seules les métaphores vives "efficaces" seraient transparentes. Lorsqu'une métaphore (comme n'importe quelle autre figure) plaît, c'est que le destinataire a cautionné la transgression des catégories évoquées ci-dessus. Qu'il trouve implicitement que cette transposition analogique-mais- transgressive propre à la métaphore est particulièrement indiquée dans le contexte. C'est dire aussi que le transfert métaphorique est perçu comme adhérant au référent. En discussion, lorsqu'il s'agit par exemple de décrire des situations très complexes, c'est souvent l'interlocuteur capable d'utiliser la meilleure synthèse métaphorique qui suscite le meilleur assentiment: "oui, c'est tout à fait cela...". Autrement dit: votre interprétation métaphorique, votre "image" est tellement pertinente, tellement vraisemblable par "modification analogique" qu'elle en est transparente, qu'elle adhère au référent. Que le figuré est plus propre que le propre...

Bref, il existe peut-être une certaine valeur d'échange de la métaphore. Un capital symbolique dirait Bourdieu. Elle passe inaperçue lorsqu'elle est transitivement communicationnelle. Par contre, elle fait

1 Pour utiliser la terminologie de F. RÉCANATI, Transparence et énonciation, Paris, Seuil, 1978. 
résistance négative dans le cas d'un usage perçu comme stéréotypé, et résistance positive dans le cas d'un usage étonnant associée à l'opacité (la "richesse" imaginative) d'un style subjectif en pleine manifestation expressive.

\section{La métaphore et son travail narratif}

Récit et métaphore peuvent donc être envisagés sous l'angle de leur affinité conceptuelle et en fonction d'un parallélisme partiel. Attachons-nous maintenant au potentiel de leurs interactions. J'envisagerai plus précisément quelques usages narratifs de la métaphore. Car celle-ci peut parfois participer activement à l'économie narrative. Les cas brièvement observés se situent surtout dans le contexte communicationnel des médias de masse véhiculant des récits en images (cinéma, bande dessinée, télévision...).

Dans beaucoup de récits, les événements (re)construits et configurés sont souvent associés à une sorte d'urgence linéaire, à une impérieuse stratégie de dévoilement dosé et progressif. L'instance narrative est responsable du cheminement vers ce nouvel équilibre qui clôturera le récit. Le narrateur est ainsi le gestionnaire du désir lectorial. Le "fil du récit": cette métaphore révèle bien la linéarité fondamentale du discours narratif cherchant sans cesse la continuité logique. Dans un tel contexte, développer des phases métaphoriques élaborées peut sembler un luxe de la narration. Surtout lorsqu'elle est associée à la description, la métaphore est souvent assimilable à un moment de répit. Telles seraient, selon Barthes, les séquences à dominante "indicielle". Phases de latence cognitive, pauvres en information, narrativement décisives, riches en connotations et en orientations affectives plus ou moins diffuses.

\section{L'espace métaphorique comme lieu de répit paradoxal}

Les moments de descriptions métaphoriques correspondraient dès lors à une sorte d'enlisement du récit. C'est le long regard métaphorique porté sur la Loire dans Le lys dans la vallée... Ceci n'est pas

1 R. BARTHES, "Introduction à l'analyse structurale des récits", in Poétique du récit, Paris, Seuil, Points, 1977. 
sans rappeler le couple récit-tableau proposé naguère par Ruiz et réactualisé par Benoît Peeters à propos de la bande dessinée. Les récits en images (films, BD,...) paraissent en effet reposer sur une tension entre récit et tableau. «Le récit qui, englobant l'image dans une continuité, tend à nous faire glisser sur elle. Le tableau qui, l'isolant, permet qu'on se fixe sur elle» ${ }^{1}$. Par leur effet de ralentissement, les métaphores permettraient de donner provisoirement priorité au tableau. Notons cependant que dans le cas des narrations iconiques, la question est plus complexe encore, vu l'essence métaphorique de la représentation par l'image figurative. On pourrait ainsi inverser les pôles du propos précédent et préciser que s'arrêter sur le tableau en renonçant provisoirement à l'urgence du récit, c'est aussi se donner le temps de découvrir et d'apprécier la dimension métaphorique de l'image... ${ }^{2}$.

Si on les considère comme des haltes ou des répits, les phases métaphorico-descriptives d'une narration participent d'une vaste déclinaison du schème tension-détente. Leur rôle narratif et pragmatique serait alors d'apaiser momentanément le lecteur-spectateur. Rôle souvent paradoxal, car le consommateur de récit sait combien ces phases de détentes au plan de l'intrigue sont annonciatrices de tensions futures.

\section{La métaphore comme lieu énigmatique}

Ceci permet de mieux saisir l'énoncé métaphorique dans son intégration narrative. En effet l'apparition de certaines métaphores dans le récit relève directement de ce que Barthes appelle le code herméneutique, c'est-à-dire les dispositifs permettant la gestion du dévoilement d'une "vérité" le plus souvent narrative. A l'extrême, une accumulation de métaphores complexes peut correspondre, ainsi que je le signalais ci-dessus, à l'articulation d'une énigme posée au spectateur. Une énigme, c'est-à-dire une tension sous forme d'un désir de trouver. Comme le signalait déjà Aristote:

1 Benoît PeETERs, Case, planche, récit, Tournai, Casterman, 1991, p. 34.

2 Je me permets de renvoyer à cet égard aux concepts de lecture optique vs. lecture haptique que j'ai développé in Ph. MARION, Traces en cases, essai sur la bande dessinée, Louvain-la-neuve, Académia, 1992. 
"L'essence de l'énigme consiste à décrire quelque chose par une combinaison impossible de mots; on ne peut y arriver en combinant simplement des mots ordinaires, mais en combinant des métaphores"1.

De façon moins intense, l'apparition ostensible d'une métaphore plus ou moins complexe au sein du récit ne peut laisser indifférent le "lecteur modèle". Placé dans le contexte culturel dominant d'un "pandéterminisme" narratif, il présuppose le caractère hautement fonctionnel de tous les éléments ordonnés par le récit. Comme l'explique $\mathrm{Eco}^{2}$, le lecteur-spectateur est un grand faiseur d'hypothèses. Et le surgissement d'une métaphore complexe lui donne l'occasion de mettre à l'épreuve sa compétence narrative. Le récepteur de récit est aussi un chercheur d'intentionnalité: où veut me mener l'énonciateur lorsqu'il me propose cette métaphore à cet instant du récit? Quelles instructions de lecture me donne-t-il? Quelle est -pour reprendre l'expression de Ducrot- la "composante instructionnelle" de ce qui m'apparaît comme une métaphore? La description métaphorique du Lys dans la vallée ("elle était le lys de cette vallée") peut m'apprendre beaucoup sur la personnalité centrale de l'hérö̈ne à laquelle elle est associée. Dans 2001, l'odyssée de l'espace, l'apparition récurrente d'un étrange "bloc monolithique" s'impose comme une intention métaphorique à décrypter si l'on veut entrer dans le récit. Quant au fameux "Rosebud" associé à Citizen Kane dès le début du film de Welles, nous pressentons (narrateur incarné et spectateur) qu'il s'agit là d'une métaphore décisive. La recherche de son interprétation mobilisera une part importante de l'énergie narrative.

\section{Mise en abyme}

S'il ne veut pas se priver d'une dimension symbolique et narrative importante, le lecteur-spectateur se doit donc d'ouvrir l'œil et de ne pas laisser passer ce genre de figure. Condition préliminaire, il doit reconnaître cette métaphore en tant que telle. C'est-à-dire qu'il doit saisir que quelque chose montre tout en dissimulant ${ }^{3}$. Sur le plan de la

\footnotetext{
1 ARISTOTE, Poétique, Paris, éd. des Belles Lettres, 1969, 1458 a 23-33.

2 Umberto Eco, Lector in fabula, Paris, Grasset, 1985.

3 Ce mouvement paradoxal de surexposition et d'occultation est au cœur de la métaphore, ce qui peut rendre cognitivement problématique l'utilisation de celle-ci, surtout lorsqu'elle fonde certains concepts ou modèles théoriques. Voir à ce sujet le
} 
communication narrative, il doit ensuite y déceler des intentions, les évaluer et produire des hypothèses interprétatives. Sa compétence de narrataire l'encourage à reconnaître et surveiller ces métaphores toujours susceptibles de simuler des événements futurs.

La métaphore n'est alors confirmée comme métaphore qu'a posteriori. Peut-être n'était-ce qu'une simple figure dépourvue de toute prétention d'anticipation symbolique, au plan de la narration. Mais le lecteur modèle doit rester attentif s'il ne veut pas "rater" de mise en abyme.

Car il s'agit bien de mise en abyme ${ }^{1}$ : le récit dévoile ses intentions événementielles par des anticipations métaphoriques. Sommersby, la récente adaptation américaine du film Le retour de Martin Guerre en offre un bon exemple. De l'aveu même de Richard Gere, l'acteur principal, tout le sens du film est contenu métaphoriquement dans une importante scène initiale du film:

“...une histoire qui élève l'être humain. On y développe le point de vue de la femme, celui de la terre et de la survie, et celui de l'homme, sa vision de l'héroïsme et de l'identité. La scène du rasage est une grande métaphore, une scène très belle et sensuelle. Jack donne le rasoir à Laurel qui a désormais le choix : le tuer ou le dénuder, se venger sur le barbu violent d'antan ou découvrir qui se cache sous les apparences. Tout est dans cette scène"2.

Cette figure métaphorique de soumission du héros repenti résume par anticipation toute l'intrigue développée dans la suite du récit. Cette "grande métaphore" constitue le noyau, le condensé emblématique de tous les développements diégétiques à suivre. Je pense à ces cercles de croissance qui apparaissent, irréguliers, sur les souches des arbres tranchés: par la variation de leur épaisseur, on peut y reconstruire l'histoire annuelle des saisons, le cycle des étés chauds et fertiles, celui des printemps maigres, incertains et assaillis par le gel. Certes, cette comparaison lyrique paraît erronée. Car il s'agit d'une trace. Or la trace est par nature métonymique: partielle, elle permet de reconstruire par contiguité logique un tout absent. Elle est

texte de J.-P. MeuniER, "Les théories de la communication comme métaphores qui se réalisent", in Recherches en communication, $\mathrm{n}^{\circ} 1,1994$, p. 71-92.

1 Lire à ce sujet G. DERĖzE et Ph. MARIon, "Le neuvième art en quelques abymes", in Images abymées, Les Cahiers du CIRCAV, n 4, Lille, Ed. Gérico, 1993, p. 169 et sv.

2 "Martin Guerre-made in USA", in Le Soir illustré du 3 mars 1993. 
l'effet ponctuel d'une cause globale à reconstruire. Mais elle permet de comprendre l'ambivalence de ces mises en abymes narratives: elles possèdent en effet une face métonymique: elles permettent, par synecdoque, de reconstituer le tout d'un récit en s'appuyant sur une partie de celui-ci. Mais -face métaphorique- cette partie présente de façon analogique les caractéristiques principales du tout. De telles mises en abyme relèvent en quelque sorte d'une synecdoque métaphorique.

La mise en abyme filmique décrite ci-dessus constitue grosso modo un noyau; le programme narratif y est condensé en une seule cellule prémonitoire. Ce côté génético-narratif peut, dans certains cas, être subodoré par le spectateur attentif. A partir d'une description ou d'une anecdote, le lecteur modèle, impénitent chercheur d'intentionnalités narratives, peut tenter de pressentir en quoi telle description ou telle anecdote pourraient constituer des métaphores des futurs développements de l'intrigue. Il se livre donc à un jeu d'hypothèses sur la suite du récit, un jeu d'extrapolations par analogie. En quoi cette scène est-elle emblématique des dévoilements narratifs à venir?

Notons immédiatement que ce regard métaphorique interne qui tenterait de saisir des microrécits ressemblant au macrorécit est loin de s'opérer uniquement lors d'une première lecture. Au contraire, ces microrécits métaphoriques constituent de puissants appels à la relecture. Le travail des métaphores y trouve même une meilleure occasion de s'épanouir, au sein d'un faisceau de mise en relation avec le récit "principal" déjà connu. Commentant la dernière case des Bijoux de la Castafiore dont il sera question ci-dessous, Peeters signale justement:

"Le livre revient sur ses propres traces, par ce nouvel effet d'auto-lecture, comme pour inciter le lecteur, en ce dernier instant, à prolonger lui-même le décryptage amorcé. Car finir ne saurait être autre chose, pour une fiction comme les Bijoux, qu'appeler à la relecture"1.

Le lecteur modèle est donc aussi, surtout peut-être, celui qui se souvient activement; celui qui est capable de "rewind". Se remémorer ce qu'il a déjà consommé du récit pour en exhumer certaines phases et leur donner un sens nouveau. Pour, notamment, leur découvrir cette portée métaphorique interne: "c'était donc ça le sens profond de la

1 B. PEETERS, op. cit., p. 137. 
scène" ou "cette scène que j'ai reçue au sens propre avait -bon sang, mais c'est bien sûr- un sens figuré basé sur une relation d'analogie..." La découverte a posteriori d'une portée métaphorique insoupçonnée est sans doute une source d'un des plaisirs du texte. Un plaisir de relecture.

\section{Les bijoux de la métaphore}

L'œuvre d'Hergé offre un espace de choix pour illustrer ce qui précède. Plusieurs épisodes des Aventures de Tintin qui semblent se dérouler dans la clarté la plus totale s'appuient en fait sur de féconds réseaux métaphoriques internes. Ainsi, même dans ses récits les plus classiques, on peut facilement repérer différentes formes du principe de la prédiction qui se réalise. Lors d'un bref épisode qui montre tous les signes de l'anecdote, du fortuit, de l'insignifiant, la narration révèle de manière voilée -et souvent de manière métaphorique- les événements de première importance qu'elle a programmés et prévoit d'actualiser.

\section{Prédiction et anticipation métaphoriques}

Dès la première planche des Sept boules de Cristal, par exemple, un étrange voyageur entretient Tintin à propos de l'inconscience des explorateurs de l'expédition Sanders-Hardmudth qui ont violé les sépultures ancestrales des Incas. "Ça finira mal toute cette histoire, Vous verrez..." (p.1). La suite prouvera que le voyageur au feutre noir avait raison. Cette première prédiction est relayée peu après -mais de manière plus métaphorique cette fois- par les propos de madame Yamilah, voyante de son état: "c'est un mal qui ne pardonne pas! La haine du dieu Soleil est terrible! ... Sa malédiction est sur lui." (p. 9). La suite du récit confirmera bien sûr cette anticipation narrative. De tels procédés ne sont certes pas rares en littérature, et même fréquents dans le roman policier. François Bussière précise à ce sujet: 
"Un texte générateur -prophétie, cryptogramme...- induit une action qui dans le récit lui succède (...). La «réalité» succède à une pré-diction qu'elle répète"1.

Ces prédictions relèvent surtout, sans doute, d'une chaîne métonymique: entre la prédiction et sa réalisation, il existe la linéarité d'un lien logique. Ainsi que je le précisais ci-dessus, une partie du récit permet de reconstituer, par anticipation hypothétique, la totalité de ce récit. Mais cette synecdoque possède un caractère métaphorique dans la mesure où elle constitue un microrécit offrant, dans l'analogie voilée de sa configuration, une image de l'évolution future du macrorécit.

Dans Les Bijoux de la Castafiore, on retrouve la même figure de la voyante qui énonce le déroulement futur du récit. La microséquence de prédiction y est cependant beaucoup plus métaphorique. Les propos tenus au Capitaine Haddock par la bohémienne diseuse de ... bonne aventure constituent en effet une anticipation, par transposition analogique, des événements à venir: "Toi faire très attention! sinon, accident!... Mais pas grave! ...Toi bientôt nouvelle voiture" Moi voir grande belle dame étrangère... Elle venir te rendre visite" (p. 4). L'“accident" sera la chute et l'entorse du capitaine. En fait de "nouvelle voiture" le Capitaine devra se contenter d'une chaise à roulettes pour invalide... A priori, la dimension métaphorique des paroles de la bohémienne ne peut être perçue que de manière rétroactive. Il faut être plus avancé dans le récit pour comprendre ce qu'est vraiment cette nouvelle voiture annoncée et pour percevoir la métaphore ironique présente dans cette prédiction. La métaphore, dans ce cas, ne transparaît qu'en re-lecture, au moins mentale. Mais il convient sans doute de nuancer, car même lors d'une première découverte de l'œuvre, la page de garde de l'album est construite sur l'image d'une triomphante Castafiore poussant la chaise roulante d'un Capitaine passablement décati. Même à la première lecture, le lecteur peut donc évaluer la portée métaphorique de la scène de bonne aventure en la reliant à cette sorte de mise en abyme initiale, présentée dans la page de garde. Celle-ci constituant en elle-même une sorte de prégénérique propre à la bande dessinée. A cela s'ajoute la probable connaissance encyclopédique propre à ce même lecteur: de mémoire de tintinophile, Haddock n'a jamais possédé de voiture personnelle, c'est donc que le sens de cette "nouvelle voiture" annoncée est à cher-

1 F. Bussière, “Arsène Lupin, homme de lettres", in Revue Europe, n571-572, cité par B. PEETERS, Les bijoux ravis, p. 15. 
cher ailleurs. C'est donc qu'il faut enclencher son regard métaphorique et chercher le "sens figuré" pour rester bien en phase avec le récit.

La suite de la prédiction de la bohémienne évoquera le vol des bijoux: "Bijoux partis!...Disparus!...Envolés!...". Ici, l'anticipation est plus diabolique encore. Impossible de deviner, à l'orée du récit, le "vol" des bijoux par la pie. Dans une sorte d'inversion du dispositif décrit ci-dessus, le lecteur prend spontanément le terme "envolés" dans son sens métaphorique. Ce qu'il ignore encore, c'est que les bijoux vont "vraiment" s'envoler et que la figure devrait être prise au sens littéral. Ou plutôt que cette métaphore si claire n'en est pas une, ou encore, qu'elle est un agent double...

Au plan de la réception, ces dispositifs métaphoriques -même lorsqu'ils sont décelables lors d'une première lecture-, n'atteignent donc leur plein épanouissement que dans l'espace d'une relecture. Comme l'énonce judicieusement Benoît Peeters:

"La prédiction est aussi une manière de donner au lecteur, dès le début du livre, toutes les cartes nécessaires. Il n'y a aucun trucage: le lecteur dispose de tous les éléments. Toujours, il aurait pu deviner la solution: il suffira qu'il entame une relecture pour immédiatement s'en rendre compte. Le suspense se nourrit de semblables défis en même temps que se renforce la réflexivité du volume et l'affirmation de sa fondamentale clôture"'.

Le fil de l'intrigue étant connu, l'attrait de la découverte -le code herméneutique- peut se reporter sur les ressources métaphoriques du récit, et singulièrement sur la richesse des anticipations par métaphores.

\section{Le mystère de l'étoile métaphorique}

La prédiction relève finalement de l'arsenal classique des procédés narratifs lorsqu'il s'agit d'organiser le déterminisme serré des récits à suspense.

"Une des fonctions d'“annonces" (...) est d'ailleurs de signaler au lecteur, à un moment où l'histoire n'a pas vraiment commencé, la proximité des véritables événements: un récit qui

1 Ibidem, p. 16. 
commence lentement est dans l'obligation de multiplier les signes de l'action qui va suivre, afin d'éviter que le lecteur ne s'impatiente"1.

Cependant, si les narrations hergéennes laissent une telle impression de cohérence, c'est sans doute aussi (surtout) grâce à la qualité des réseaux métaphoriques et symboliques qui tressent le récit, audelà de ces lieux codifiés que constituent la prédiction initiale. En guise d'illustration, je me servirai d'une cuvre plus ancienne: L'étoile mystérieuse.

Tous les lecteurs se rappellent de l'oppressant début de l'histoire. Une étoile s'approche dangereusement de la Terre, l'angoisse croît dans les rues de la ville plongée dans une blafarde pénombre bleutée, tandis que la chaleur devient de plus en plus suffocante. Pour qui connaît sa coutumière ignorance des dangers, Tintin lui-même paraît étrangement inquiet. Il se rend à l'observatoire pour en savoir plus. Sur le conseil du Directeur -"le spectacle, dit-il, en vaut la peine"Tintin jette un coup d'œil dans le télescope. Horreur! L'étoile est habitée d'une immense araignée "aux pattes velues". "Elle est énorme! Énorme! ...", s'exclame Tintin.

Ce passage constitue sans doute une prédiction, mais cette fois, beaucoup plus riche et complexe, parce qu'elle échappe aux lieux habituellement réservés à cet effet. Et surtout parce qu'elle utilise idéalement les ressources spécifiques du média BD. Au plan de la monstration, tout d'abord, 1'image" de l'énorme araignée perçue par Tintin est bien sûr l'anticipation métaphorique de l'araignée énorme à laquelle il sera "vraiment" confronté à la fin du récit. L'aérolite sur lequel il se trouve alors contient en effet du "calisthène" qui a la propriété de faire grossir de manière accélérée les éléments vivants qui entrent en contact avec lui: le trognon de pomme donnera en quelques heures des pommiers énormes, la chenille deviendra un immense papillon, idem pour les champignons. Et surtout, cette "petite" araignée échappée de la musette de Tintin deviendra le monstre que l'on sait.

Cette figure de l'araignée va donc unir le début et la fin de l'album. D'abord affirmée brutalement, sa démesure se trouve bientôt remise dans le cadre d'une méprise et d'une illusion d'optique. Elle constitue une erreur d'évaluation du "réel" commise par le héros. Mais de l'erreur qu'elle était, elle va accéder au statut de "vérité" et se hisser

1 Ibidem, p. 13. 
au premier degré de la narration-représentation. Tellement vraie que Tintin en personne est obligé de fuir pour lui échapper. D'image fantasmée, elle s'est transformée en danger réel.

Sous sa forme d'illusion d'optique, l'araignée du début est, comme toute métaphore, basée sur une erreur organisée, une transgression codée. Mais cette erreur est, de façon prédictive, porteuse de futures analogies. Alors que le lecteur croyait pouvoir la laisser au second degré de représentation fictionnelle, voilà qu'elle finit par s'imposer au premier degré, manifestant ainsi la force métaphorique de sa première apparition et renforçant la clôture homogène du récit. Outre ces deux moments clefs, l'araignée refera une autre apparition importante à l'occasion d'un cauchemar de Tintin: il voit le prophète Philippulus annoncer "le châtiment" en déroulant l'image d'une immense araignée. Parallèlement à l'illusion d'optique qui précède, le monstre est maintenu, en abyme, dans un deuxième degré de fiction. Voire même un troisième degré, car l'image brandie de l'araignée est elle-même intégrée dans le cauchemar de Tintin. Comme si l'effet d'horreur s'éloignait du premier plan fictionnel (c'est-à-dire celui ou l'effet de réel tente d'être le plus indiscutable) et s'usait en sédimentations successives, la rendant de plus en plus inoffensive par son enfouissement en abyme. Avant, bien sûr, de ressurgir avec fracas.

Notons que cette construction manifeste un exemplaire mouvement de relais entre le texte et l'image. Ainsi, la frayeur de Tintin face à l'araignée-illusion s'est d'abord jouée exclusivement au plan de la monstration: l'image de l'araignée sur l'étoile que le lecteur découvre avec lui. Puis elle ne tarde pas à se prolonger au plan du langage verbal et du dialogue (les commentaires effrayés de Tintin). Ce passage au langage va lui-même permettre un effet rhétorique. Sceptique face aux réactions de Tintin, le professeur Calys s'inquiète: "Une araignée! Est-ce que vous n'en avez pas une dans le plafond?" Par ce jeu de mots, le motif de l'araignée glisse ainsi vers une autre dimension métaphorique, spécifiquement verbale et conversationnelle. Après le passage du "prophète", avec ses images en abyme évoquées plus haut, mais juste avant le climax du face à face dramatique sur l'aérolite, l'araignée ressurgira encore dans le discours de Tintin. Un microrécit d'anticipation où le héros se fait narrateur de son propre cauchemar. En prenant une maigre collation alors qu'il est seul sur l'îlot mystérieux, Tintin se souvient en effet du prophète Philippulus: "Et ce cauchemar dans lequel, il me disait, menaçant: "Le châtiment! Aha!... Voilà le châtiment!...»Et le châtiment, c'était une araignée, une 
énorme araignée. Brrr! J'ai encore froid dans le dos quand j'y pense..." (p. 50) Le processus d'enfouissement apparent du potentiel métaphorique d'anticipation analogique est particulièrement actif ici. Dans cette scène Tintin ajoute un tour de représentation (une "sécurité fictionnelle") supplémentaire en reconfigurant, dans un récit-souvenir, l'araignée du cauchemar qui elle-même n'y figurait que représentée sur la feuille de papier déroulée par Philippulus. La stratégie narrative tient du paradoxe: reconvoquer pour le lecteur la terrifiante araignée tout en renforçant l'épaisseur de sa fictionnalité, de sa "fausseté". C'est, bien sûr, pour mieux garantir l'effet de la terrifiante révélation métaphorique imminente: la fausse araignée gigantesque du début était donc l'image fidèle de celle que nous découvrons avec Tintin. Cette araignée qui n'était qu'une illusion, qu'un cauchemar, qu'une image dans l'image, qu'un récit dans le récit, va tout à coup devenir "vraie", c'est-à-dire partager le même espace diégético-fictionnel que le héros.

Dans L'étoile mystérieuse, les dispositifs de prédiction métaphorique sont donc présentés sous le mode de l'illusion, du trompe-l'œil, de la mise en abyme. Voire même d'une vision fantasmatique partagée par le lecteur grâce aux dispositifs propres à la $\mathrm{BD}$ : c'est dans cette direction que se développent les intéressants commentaires de Jacques Samson, à propos de la fameuse scène de l'observatoire:

“...la réalité donnée à voir se moule d'après les perceptions erronées de Tintin. (...). Ainsi, par le jeu d'une substitution dans l'ordre du paradigme, la vision lectorielle devient l'objet d'un transfert interprétatif: aux yeux du lecteur, la vue de l'araignée est donnée en lieu et place du regard de Tintin. (...). Ici la bande dessinée est mise en scène et mise en acte du fantasme: elle mêle à la fois la vision fantasmatique (la vue de l'araignée géante) et le regard fantasmé (le point de vue de Tintin). Le procès métaphorique à l'œuvre dans ces images lie très étroitement le voir et le savoir, de façon à placer Tintin et le lecteur exactement sur le même pied"'.

Mais ces visions erronées, ces illusions vont se hisser au niveau de la "réalité" fictionnelle, renforçant le vraisemblable de celle-ci. Parallèlement, dès le moment où le lecteur a découvert leur sens propre, ces mêmes illusions vont revêtir le sens figuré d'anticipation,

1 J. SAmson, "Images d'angoisse: Tintin, l'araignée et le champignon", in Urgences, n³2: Lectures de bandes dessinées, Ottawa, 1991. 
de métaphores prédictives. Une telle mise en abyme par métaphores construit un important effet de tressage sémantique et symbolique. Le trait récurrent de l'araignée grossie se déploie par affleurements métaphoriques anticipant l'apparition finale de l'Épeire Diadème. En soustexte, le réseau progresse parallèlement mais aussi et surtout solidairement au récit. Avec l'utilisation de cette métaphore-illusion, le lecteur est en quelque sorte préparé négativement à la scène de l'aérolite: l'anticipation analogique est en effet frappée de non-vérité, certes, mais elle s'est cependant imposée dans l'esprit du lecteur (et de Tintin) avec la prégnance d'une terrifiante vision fantasmatique qu'on tente d'oublier ou de relativiser par un récit distancié. Dans un mouvement étrangement solidaire, la représentation fantasmatique va devenir "réalité" et la métaphore, tout en se révélant ou se confirmant, va -paradoxalement- se défaire de sa dimension métaphorique pour être prise au premier degré, dans la littéralité de son expression. Et la narration de tirer désormais l'énergie de ses nouveaux développements dans -pour plagier Fresnault-Deruelle'- une "image prise au mot".

Mais la figure de l'araignée n'est sans doute que la manifestation spectaculaire d'un motif plus vaste qui sous-tend tout l'album: le grossissement. Omniprésent ce processus abstrait trouve bien sûr de multiples incarnations métaphoriques: à côté de l'araignée sur la lunette du télescope, on trouve aussi l'étoile qui n'arrête pas de grandir aux yeux des humains effrayés ("pas de doute elle a encore grandi", répète Tintin). Quand à l'aérolite, j'ai déjà évoqué sa capacité de provoquer de l'accroissement accéléré.

C'est dire que ce motif lui-même pourrait être interprété comme métaphore emblématique plus vaste encore: une transposition par équivalence du propre fonctionnement du récit de BD. Ainsi, sur l'aérolite, le grossissement est spatio-temporel. Les dimensions hors normes relèvent de l'espace, et la croissance, du temps. N'est-ce pas là une métaphore de la BD, ce moyen d'expression qui se construit sur la confusion organisée des deux paramètres: de l'espace qui fait croire au temps et du temps qui ne peut s'y matérialiser que dans l'espace? Cette pomme qui devient un immense pommier en quelques heures de diégèse, c'est la métaphore du "syndrome de Nemo"2. C'est-à-dire, entre

1 P. Fresnault-Deruelle, Les images prises au mot, Paris, Edilig, coll. Médiathèque, 1989.

2 L'expression est de A. Altarriba, "Propositions pour une analyse spécifique du récit en bandes dessinées", in Bande dessinée, récit et modernité, Colloque de Cerisy, Paris, Futuropolis, 1988. L'auteur utilise le nom du célèbre héros de Mac 
autre, cette extrême facilité que possède la $\mathrm{BD}$ de représenter les phénomènes figuro-narratifs de métamorphose. Cheminant de cases en cases, et d'ellipses en ellipses, toutes les transformations y sont "ontologiquement" possibles. Là où la continuité des images cinématographiques se heurte à la contingence d'un flux évolutif. (Le vidéoclip tente sans doute de contrarier cette continuité spatio-temporelle du film, qui est aussi garante de la prégnance de son illusion). Dans le même esprit, Peeters voit dans ce motif de l'accroissement la métaphore de l'activité narrative elle-même:

"A les bien étudier en effet, il apparaît que les lois du calystène ressemblent étrangement à celle de la narration: le grossissement des objets est une parfaite métaphore de l'amplification des éléments initiaux, de ce passage du microcosme au macrocosme qui est le fin mot de l'album. Les lois de l'étoile mystérieuse sont celles de L'Étoile mystérieuse"l.

\section{Moulinsart et ses métaphores}

Cette dimension emblématique où la narration se construit tout en se nourrissant de ses propres mises en abyme, tout en utilisant à des fins diégétiques les reflets métaphoriques de son propre fonctionnement, cette dimension, donc, Hergé l'a développée au plus haut point dans Les bijoux de la Castafiore. Cette aventure qui n'en est pas vraiment une et n'en finit pas de ne pas commencer, cette histoire qui est l'aventure d'une difficile mise en histoire. Les métaphores filées y sont particulièrement actives et porteuses de narrativité. Je n'en signalerai ici que quelques-unes ${ }^{2}$ parmi les plus importantes.

D'emblée, l'aventure se place sous le signe de l'oiseau. Première case, en haut et à l'avant-plan, au-dessus du Capitaine et de Tintin en promenade, la mise en évidence d'une pie accrochée à une branche. Et

Cay pour désigner le don "naturel" de la BD de jouer de la métamorphose des personnages: "Alors que le récit littéraire se nourrit largement des changements survenant à l'intérieur d'un personnage, la bande dessinée, elle, comme jeu de formes et de surfaces, insistera plutôt sur les modifications de l'apparence." (p. 35 36).

1 B. PEETERS, Les bijoux ravis, op.cit., p. 13.

2 Pour une analyse plus complète de cette cuvre, je ne peux, une fois encore, que renvoyer au travail accompli par Peeters dans Les bijoux ravis, op. cit. 
l'on sait le rôle qu'elle jouera dans le vol "réel" des bijoux. Cette présence des oiseaux ne cessera de hanter tout le récit. Et surtout, de contribuer à son élaboration. Ainsi, par exemple, cette scène extraordinaire où Tintin, longuement embusqué dans le grenier ne surprend qu'une chouette... Cette même chouette qui le fera plus tard sursauter, lui Tintin, alors qu'il se laissait bercer par les chants nocturnes des Tziganes. Sur le plan verbal la Castafiore est en outre désignée souvent par son appellation... métaphorique: "Bianca Castafiore! Ha! Ha! Ha! ce cher Rossignol milanais" (p. 5) s'exclame un Capitaine encore inconscient du cataclysme qui le menace. Lorsqu'elle fait irruption à Moulinsart et qu'elle surprend le Capitaine tout en lui cachant les yeux de la main, son premier mot est: "coucou"...

Dans ses bagages, elle porte un cadeau personnalisé pour le vieux loup de mer: Coco, le perroquet. Le volatile va jouer un rôle de premier plan dans la suite de l'histoire. Au départ lié à la Castafiore par adjonction (par un lien de type logico-métonymique: l'invitée et son cadeau) le récit va progressivement le faire entrer dans un espace métaphorique. Petit à petit, en effet, le Perroquet s'impose comme substitut analogique de la Castafiore. Une équivalence de type paradigmatique s'affirme de façon de plus en plus claire sur le fil syntagmatique du récit. Parcourons quelques étapes de cette évolution vers la condensation métaphorique. Tout d'abord, au moment du cadeau, la diva le présente comme "le plus fidèle des compagnons" (p. 9). Un compagnon, sans doute, mais bien agaçant: ne quittant pas un instant le Capitaine impuissant et immobilisé, il n'arrête pas de l'agresser en "paroles" ("allô, j'écoute!", etc.) et surtout en morsures diverses (le doigt, le nez). Page 14, l'association entre la cantatrice et l'oiseau se fait plus explicite: espérant trouver le répit dans le sommeil ("il finira par me rendre fou! ... Heureusement, c'est bientôt l'heure d'aller dormir: comme ça au moins j'en serai débarrassé pour toute la nuit"), le Capitaine fait un cauchemar. Sur une scène d'opéra, un perroquet habillé en Castafiore -ou une Castafiore aux allures de perroquet-y va de son légendaire "Ah! Je ri-i-i-is". Dans le public composé de perroquets en smoking qui le dévisagent avec un lourd mépris, un Haddock aussi nu que rouge...

C'est littéralement la genèse d'une métaphore qui se donne à voir ici. En condensant ainsi les attributs de l'une et de l'autre, le monstrateur-narrateur offre au lecteur la clef d'un passage à la figure. Il lui montre l'opération de substitution analogique comme si elle était surprise dans sa genèse transformationnelle, dans cette temporalité 
élidée que je signalais au début de cet article. Il lui confirme aussi le programme métaphorique qu'il a mis en place. Dorénavant, plus de doute, le perroquet équivaut à la Castafiore et peut se substituer à elle: non seulement le Rossignol milanais et le perroquet se ressemblent, mais ils sont de plus investis des mêmes fonctions narratives: agressions et harcèlements divers sur le Capitaine. Cette confirmation métaphorique par mise en analogie se reproduira quelques pages plus loin lors de la séance de télévision couleur organisée par le Professeur Tournesol: la Castafiore "transformée" par les problèmes de mise au point technique ressemble à s'y méprendre à un oiseau (p. 49). A plusieurs autres endroits du récit, la proximité et/ ou l'équivalence entre la Castafiore et le perroquet seront d'ailleurs régulièrement rappelées pour que se conforte -tout en se matérialisant provisoirement- la comparaison sous-entendue nécessaire à l'épanouissement métaphorique (cf. par exemple p. 41: le magazine Tempo di Roma montrant et titrant: "La diva e il pappagallo").

Remarquons aussi cette singularité étonnante: toutes ces traces d'un travail et d'une intentionnalité métaphoriques apparaissent dans des lieux de fiction au second degré, ou plus exactement, dans ces lieux posés comme en retrait au plan de l'effet de vérité-réalité principal. Comme dans L'étoile mystérieuse. Cauchemars, télévisioncouleur dans ses premiers balbutiements, magazines à sensation...: les clefs métaphoriques nous sont en effet proposées dans un espace de représentation décalé. Un espace en abyme, un espace pointé comme artefact métaphorique, dans la cohérence vraisemblable de l'artefact de base: celui de la fiction principale qui précisément se doit, à coup de cohérence, d'occulter sa fictionnelle facticité. En fait, la stratégie narrative est double: renforcer par différence le vraisemblable du récit principal tout en laissant germer et s'engouffrer la prolifération métaphorique qui finit par envahir tout le récit.

Cette chaîne métaphorique de l'oiseau, couronnée par la figure du perroquet, entre bien sûr en interaction avec d'autres chaînes. Ainsi, le motif de l'agression lié à la féminité parcourt lui aussi tout l'album avec de multiples incarnations métaphoriques. Dès la morsure initiale de la petite Miarca -c'est la première fois que l'on peut voir la couleur du sang dans les aventures de Tintin, c'est aussi la morsure d'une petite fille habillée en rouge opérée sur la main d'un vieux loup de mer qui lui a précisé la case d'avant: «on ne va pas te manger tout de même!'...- les agressions se multiplient sous toutes leurs formes: les gammes du pianiste, les cris, les piqûres de guêpes, les autres 
morsures déjà évoquées. Inutile de préciser à quel point les éléments décrits ci-dessus stimulent idéalement les lectures psychanalytiques ${ }^{1}$ : un univers masculin incapable d'accéder à l'aventure qui pourtant l'avait toujours défini. Un univers livré à la clôture, au vase clos d'une aventure qui refuse de s'épanouir, et soumis, impuissant, à l'irruption autoritaire de la féminité. Et quelle féminité, une petite fille espiègle et mordeuse relayée par une Diva non désirée mais dont on doit bien respecter l'autorité. Une Diva doublée métaphoriquement d'une équipe de volatiles chargés de concrétiser l'agression (la castration implicite): le perroquet, les guêpes. Avec une telle lecture, tout l'album devient bien sûr la métaphore d'une structure globale: les saccages causés par l'irruption d'une féminité castratrice dans un paysage obstinément masculin.

Plus généralement, tout le récit se présente comme un jeu sur la figure, une oscillation ludique entre la fiction et la "non fiction", un va-et-vient entre le sens propre et le sens figuré. Ce qui était métaphorique ou imagé dans les autres albums tend à envahir le premier degré: les bijoux ne sont plus qu'une simple parole de "l'Air des bijoux", ils participent à la diégèse; le sobriquet "Rossignol milanais" tend à se littéraliser chez la Castafiore qui ressemble de plus en plus à un oiseau, etc. Inversement, ce qui fonctionnait au premier degré semble glisser vers un sens second: le motif du voyage et de l'exotisme, si central dans les autres récits de la série, se trouve transféré vers des personnages secondaires (les romanichels), voire remisé dans la formule littéraire qui les nomme: les gens du voyage. Désormais, l'exotisme n'existe plus que métaphorisé. Ces gens du voyage deviennent ainsi le reliquat métaphorique -la sublimation poétique- de cet exotisme jadis si pleinement vécu par les personnages et le lecteur. Alors que tous les autres albums reposent sur l'aventure vécue sans recul, celle-ci devient même un sujet de contemplation nostalgique -Tintin s'émeut à distance en écoutant le chant des gens du voyage- ou un simple jeu sans conviction.

Sur le plan de la communication narrative, le processus de lecture lui-même est attiré dans un piège. Si les personnages ne vivent que des soubresauts de récit, s'ils tombent et se trompent, le lecteur subit un sort comparable. Comme Tintin embusqué pour rien dans le grenier du château, le lecteur tintinophile attend vainement que quelque chose se passe "vraiment". Le récit hésitant devient alors le

1 Lire notamment les ouvrages de Serge Tisseron. 
miroir de son propre désir herméneutique. Comme Hitchcock, Hergé déjoue les horizons d'attente du lecteur-spectateur. Mieux: il les déjoue en les aiguillant sur la voie du métalangage. Il les met en abyme. Songeons à cette fameuse marche cassée sur laquelle tous les personnages -excepté la Castafiore!- viennent trébucher. Ces chutes répétitives et obsédantes peuvent se lire comme une métaphore des multiples culbutes de la mise en intrigue. Cette intrigue que le lecteur croyait enfin naissante. Ces vols planés ne seraient-ils pas la métaphore des frustrations et des enthousiasmes narratifs brutalement avortés d'un lecteur trop naîf qui lirait Les bijoux de la Castafiore comme Tintin en Amérique...

Dans Les Bijoux de la Castafiore, le jeu métaphorique acquiert une dimension d'emblème, dans le sens d'une désignation métaphorique d'un art et de ses processus. Ce qu'explique Peeters à propos du génial Vertigo d'Hitchcock pourrait sans doute être transposé ici:

"Le récit de Vertigo est d'abord l'histoire d'une entrée dans l'illusion (...) Plus subtilement encore que Rear Window, Vertigo propose donc une métaphore de la situation cinématographique, de ce rapport entre auteur et spectateur qui pousse le second à vouloir entrer de plein pied dans la fiction, à vouloir donner corps à un simple trompe-l'œil. Vertigo, c'est l'histoire d'un homme qui ignore ce que c'est qu'une image, c'est la prise au sérieux de l'illusion référentielle"1.

Ce travail autoréférentiel de l'œuvre qui ne peut s'accomplir que dans un miroir a certes connu son heure de gloire avec l'école du nouveau roman ou du nouveau cinéma. Cherchant à légitimer théoriquement ce mouvement, Ricardou précisait que "les grands récits se reconnaissent à ce signe que la fiction qu'ils proposent ne sont rien d'autre que la dramatisation de leur propre fonctionnement"2. Cette formule convient parfaitement aux Bijoux de la Castafiore. Mais il me semble qu'elle n'est pas loin de s'appliquer à l'œuvre d'Hergé toute entière. Avec une différence de taille, cependant: face aux œuvres qui poussent beaucoup plus loin les récurrences, les figures profondes et les réseaux métaphoriques, les récits hergéens privilégient toujours une linéarité narrative apparente. Dès lors qu'il se

1 Benoît Peeters, Hitchcock, le travail du film, Paris, Les impressions nouvelles, 1993, p. 47-48.

2 J. RICARDOU, Problèmes du nouveau roman, Paris, Seuil, 1967, p. 178. 
joue dans l'ombre tout en tirant secrètement plus d'un fils du spectacle narratif, le travail des métaphores et figures ne me paraît que plus important à épingler. Car l'intensité des effets varie selon le contexte. Placés dans l'urgence d'un dévoilement narratif, ces effets ne s'imposent qu'avec un effort méritoire. La transparence linéaire de l'intrigue, doublée par la clarté graphique, entre alors en confrontation dynamique avec l'opacité circulaire d'un réseau métaphoricoemblématique et d'un sous-sol métaphorique. Comme le précise encore Peeters:

"le choc de l'effet paradigmatique étant pour ainsi dire proportionnel à la résistance de l'axe syntagmatique, ces échos et ses rimes, ces figures et ces emblèmes ont un impact extrême dans des récits aussi tendus..."

\section{Vers une médiatique de la métaphore}

Les occasions de complicité entre la métaphore et le récit ne semblent donc pas manquer. Les formes en sont diverses. Et variables les intensités interactives. Les exemples commentés ci-dessus montrent combien la métaphore peut stimuler la gestation narrative et conforter cet intense "déterminisme" nécessaire à l'épanouissement d'une intrigue classique. Mais de façon plus fondamentale, les stratégies narratives les plus habiles ne se mesurent peut-être que dans l'art qu'elles manifestent à tresser des réseaux métaphoriques internes. L'étoffe d'une narration me semble même se tisser surtout dans l'épaisseur cohérente de son travail métaphorique. Cohérente, parce qu'en profonde intelligence avec la construction narrative. Le travail du récit ne ferait alors que mettre à profit systématiquement, dans son espace clos de représentation, cette propension à l'autoréalisation que semblent manifester, plus généralement, les métaphores de la vie quotidienne ${ }^{2}$.

Solidaire du développement narratif, la dimension métaphorique d'un récit constitue aussi une ouverture au pluriel et aux spirales d'un métalangage. De plus, lorsque les réseaux métaphoriques prennent

1 Benoît PeETERs, op. cit., p. 60-61.

2 Voir George LAKOFF et Mark JOHNSON, Les métaphores dans la vie quotidienne, Paris, Edit. de Minuit, 1983. Lire aussi J.-P. Meunier, "Les théories de la communication...", op. cit. 
une importance emblématique, ils peuvent aussi engendrer un espace relationnel de métacommunication. La métaphore ne servirait donc pas qu'à stimuler intérieurement la narration. Sa capacité propre de construire l'écart figural (rhétorique), son mécanisme définitionnel de transposition analogique, la rendraient apte à refléter spéculairement l'état d'une relation au lecteur, elle serait alors le lieu privilégié d'une relation virtuelle. Au plan de la réception, comprendre ou pressentir une métaphore emblématique, c'est aussi contempler et saisir dans et par le récit son mode de participation à ce récit. Au sein du message, le contrat fictionnel implicite ne peut affleurer que dans l'emblème. Par son essence de miroir -mais de miroir forcément déformantl'écart figural postule une anamorphose. Le jeu métaphorique finit par obliger le lecteur spectateur à ne plus se prendre au jeu. Ou à se regarder, dans une sorte de demi-conscience, en flagrant délit d'acceptation de se laisser prendre à ce jeu.

La perspective paradigmatique est forcément plus apte à tendre des miroirs et à mettre en abyme. Échapper à la transparence du récit, c'est laisser s'immiscer le métalangage. Mais c'est aussi une acceptation du murmure croissant des faisceaux métaphoriques.

Une approche des liens tissés entre métaphore et narrativité ne peut, selon moi, faire l'économie d'un autre aspect trop long à développer ici: celui du média, ou du moyen d'expression choisi. A travers le travail d'Hergé observé ci-dessus, on a pu en effet sentir l'importance décisive d'une prise en considération des caractéristiques propres au média bande dessinée. Pour s'épanouir pleinement, narration et réseaux métaphoriques doivent exploiter au mieux les capacités propres au matériau d'expression qu'elles utilisent: contiguité et consécution d'images dessinées immobiles, liens intenses tissés sur l'espace de la page, suites des planches... toutes ces composantes fondamentales de la $\mathrm{BD}$ permettent par nature un certain développement narrativo-métaphorique. Cette réflexion pourrait être située face à un nouveau champ disciplinaire que j'ai suggéré ${ }^{1}$ d'appeler la médiatique narrative.

Se nourrissant des questions de transécriture et de transsémiotique, cette discipline se propose d'étudier la rencontre d'un projet narratif -d'un récit non encore fixé dans sa matière d'expression définitive- avec la force d'inertie ou la force de gravité d'un média donné. Pour être communiqué, un substrat événementiel réel ou

1 Philippe MARIon, Traces en case, op. cit., p. 278. 
imaginaire doit, en effet, utiliser un moyen de médiation qui lui permettra de trouver sa configuration. C'est par ce corps à corps avec la narrativité ontologique d'un média que pourra s'exprimer la cohérence d'une mise en intrigue. Lorsqu'on le fait "tourner à vide", un média ne tarde pas à révéler son potentiel expressif et narratif propre. Ainsi, le cinéma comme la bande dessinée ont par essence un je-ne-sais-quoi de narratif dès lors qu'ils se définissent par la manière dont ils agencent et font se succéder les images. La manière aussi dont ils stimulent la possibilité d'introduire un principe de transformation dans cette succession. Charge à ce potentiel narratif intrinsèque d'interagir alors avec un contenu-récit extrinsèque. Chaque projet narratif pourrait donc être considéré dans sa médiagénie 1 . Fables et histoires auraient ainsi la possibilité de se réaliser de manière optimale en choisissant le partenaire médiatique qui leur convient le mieux. Tel serait le cas des Aventures de Tintin. Conçues pour et au cour de la dynamique graphique de la bande dessinée, celles-ci sont pratiquement impossibles à adapter.

Dans le même esprit, il me semblerait passionnant d'étudier la manière dont chaque média rencontre, actualise, stimule ou recrée le dispositif métaphorique. Les modalités selon lesquelles la métaphore, dans sa figurale spécificité, peut entrer en réaction avec l'expressivité ontologique d'un média. Tel serait l'objet d'une médiatique de la métaphore.

1 Ibidem, p. 278 et sv. 